\title{
Spectral profile cues in comodulation masking release
}

\author{
Emily Buss $^{\text {a) }}$ \\ Department of Otolaryngology/Head and Neck Surgery, University of North Carolina School of Medicine, \\ Chapel Hill, North Carolina 27599
}

(Received 8 July 2009; revised 16 March 2010; accepted 17 March 2010)

\begin{abstract}
Previous work on spectral shape discrimination has shown that detection of a level increment in one tone of a tonal complex is dependent on spectral position, with thresholds forming a "bowl" pattern for components spanning 200 to $5000 \mathrm{~Hz}$ [Green, D. M., (1988). Profile Analysis: Auditory Intensity Discrimination (Oxford University Press, New York)]. The current study examined whether a similar bowl occurs for comodulation masking release, a paradigm in which dynamic spectral cues could be used to detect an added signal. Maskers were logarithmically spaced 15-Hz-wide bands of noise. The signal was a tone or a copy of the on-signal masker band. When the masker was composed of one or more random bands, thresholds were relatively consistent across frequency. When the masker was a set of comodulated bands, thresholds for both signal types formed a bowl, but the minimum threshold occurred at a higher signal frequency for the tonal than for the narrowband noise signal. Results for additional conditions indicate that spectral effects depend on both absolute frequency and relative frequency of the signal within the masker. Data collected with flanking maskers presented contralateral to the signal and on-signal masker indicate that peripheral effects may play a role in threshold elevation at high signal frequencies with narrowband noise signals. (c) 2010 Acoustical Society of America. [DOI: 10.1121/1.3397410]
\end{abstract}

PACS number(s): 43.66.Ba, 43.66.Dc [BCM]

Pages: $3614-3628$

\section{INTRODUCTION}

Comodulation masking release (CMR) is the detection advantage associated with masker envelope coherence across frequency. In a typical CMR experiment, detection thresholds are measured for a pure-tone signal in each of three stimulus conditions: with a single narrowband masker centered on the signal frequency (on-signal), with a set of random narrowband maskers (random), or with a set of coherently amplitude-modulated narrowband maskers (comodulated). Thresholds are typically lower in the comodulated masker than in either the on-signal or the random masker (Hall et al., 1984), a finding frequently interpreted as reflecting across-channel processes. There has been no shortage of theories about the cues underlying CMR, with many focusing on the change in envelopes across frequency with addition of a pure-tone signal (Buus, 1985; Hall, 1986; Richards, 1987; van de Par and Kohlrausch, 1998). For example, Buus (1985) proposed that masker fluctuations in channels adjacent to the signal frequency cue the auditory system to give more perceptual weight to the output of the channel centered on the signal during epochs of low masker energy. An emphasis on signal energy coincident with masker envelope minima could also result from acrossfrequency envelope comparison following basilar membrane compression (Buus et al., 1996). Other reports have identified possible within-channel cues associated with adding a pure-tone signal to a set of comodulated narrowband maskers (Schooneveldt and Moore, 1987; Berg, 1996; Verhey et al., 1999).

\footnotetext{
a) Author to whom correspondence should be addressed. Electronic mail: ebuss@med.unc.edu
}

In an early demonstration of CMR, Hall et al. (1984) suggested that some of the processes underlying the detection advantage conferred by masker comodulation could be the same as those studied in the profile analysis paradigm (see also Moore and Glasberg, 1987; Fantini and Moore, 1994a; Eddins, 2001). In a typical profile analysis experiment, the standard stimulus is a complex composed of equalamplitude pure tones, and the signal stimulus is characterized by a level increment to one of these tones. A level rove is imposed on the stimuli to prevent absolute level at the signal frequency from serving as a reliable cue, encouraging the listener to use the relationship of levels across frequency to detect the signal (Green, 1988). Green and Nguyen (1988) showed that listeners could make use of a spectral profile cue even if the stimuli were sinusoidally amplitude modulated at a low rate $(<40 \mathrm{~Hz})$, provided that the modulation was coherent across frequency. Similar results were reported by Hall and Grose (1988), who concluded that masking release could be based on across-frequency envelope decorrelation or on a change in the spectral profile. A number of subsequent studies examined the use of detection cues available in typical CMR and profile analysis paradigms, as well as hybrid conditions with a combination of envelope and longterm spectral cues (Fantini et al., 1993; Fantini and Moore, 1994a, 1994b; Bacon and Smith, 1996; Richards and Lentz, 1998; Eddins, 2001). Results of these studies are consistent with the hypothesis that CMR and profile analysis are based on the same cues and perceptual processes under some conditions.

Figure 1 illustrates some of the cues present in a typical CMR condition, where the signal to be detected is a pure tone, and a condition intermediate between CMR and profile analysis, where the signal is a copy of the on-signal noise 

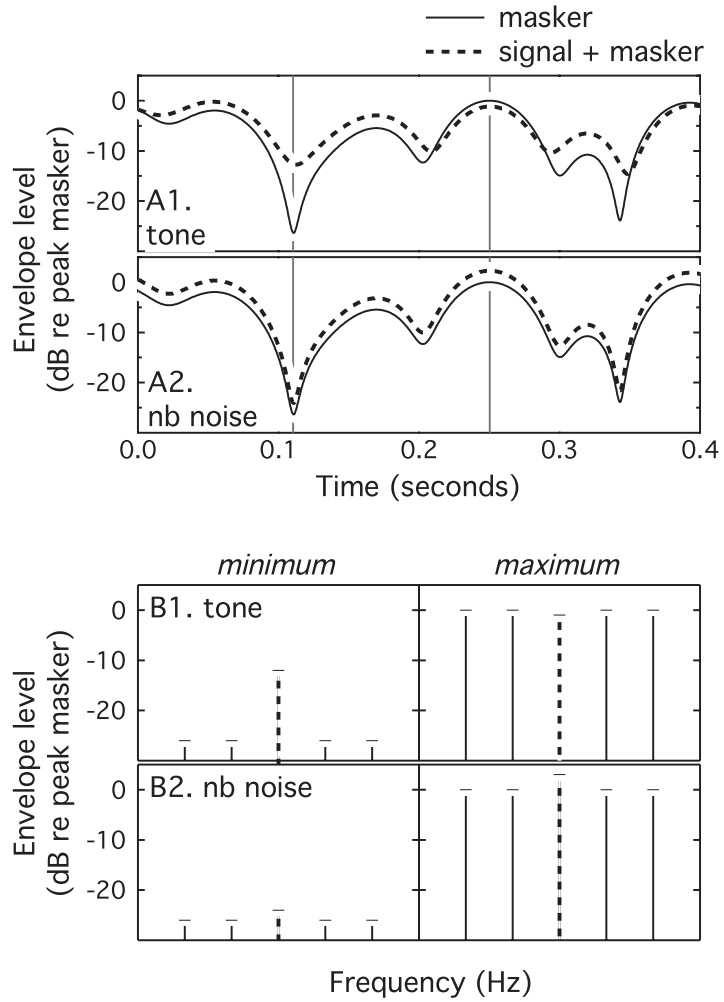

FIG. 1. Schematic of stimulus features associated with detection of a signal in comodulated maskers in the time domain (A) (top) and the frequency domain (B) (bottom). The top portion of each panel is for a pure-tone signal, and the bottom portion is for a signal that is a copy of the narrowband of noise to which it is added. The vertical lines in the time-domain panels indicate a local masker envelope minimum and a maximum. Frequencydomain panels show short-term power spectra associated with those two envelope events.

band to which it is added. The top two panels show the envelope for a masker (solid line) and a signal-plus-masker (dashed line), plotted in $\mathrm{dB}$ re peak masker level. Vertical lines indicate a masker envelope minimum and an envelope maximum. For the pure-tone signal, the envelope difference resulting from the interaction of the signal and masker is greatest during the masker minima (A1). In contrast, when the signal is a copy of the masker band to which it is added, the envelope difference is constant over time (A2). These same stimulus features are illustrated in the frequency domain in the bottom two panels of Fig. 1. Adding a pure-tone signal to a set of comodulated masker bands causes the short-term spectrum to deviate from flat, with a prominent spectral peak at the signal frequency during the masker modulation minima (B1). When the signal is a copy of the on-signal band to which it is added, there is a constant increment at the signal frequency regardless of the masker envelope value (B2).

Characterizing the cues underlying CMR in terms of "across-frequency envelope difference cues" or as "dynamic spectral cues" is largely equivalent from a stimulus perspective, as illustrated in Fig. 1. However, there are several potential advantages to describing cues in CMR and profile analysis in common terms. If the behavior highlighted by these paradigms results from a common mechanism or feature of auditory processing, then advances in modeling could be shared across paradigms. For example, Richards and Lentz (1998) adapted Durlach's channel model for spectral shape discrimination (Durlach et al., 1986) to characterize the effects of short-term changes in spectral profile. This approach could also be valuable in modeling CMR to the extent that CMR is based on short-term spectral cues. On the other hand, if behaviors revealed by the CMR and profile analysis paradigms differ in fundamental ways, then such a distinction could have important implications for the processing of stationary as compared to dynamic spectral cues.

Some CMR data may be most parsimoniously described in terms of dynamic spectral cues. A recent study by Buss et al. (2009) showed that the effects of reducing envelope coherence depend on the unique envelope features of the on-signal masker as compared to the flanking maskers. In one set of conditions, detection threshold for a pure-tone signal was measured in the presence of amplitude-modulated tonal maskers. Modulation was either at a fast or a slow rate. The fast envelope was a $20 \mathrm{~Hz}$ raised cosine. The slow envelope had a $10 \mathrm{~Hz}$ rate, with each period consisting of a 50 ms steady state followed by one period of a $20 \mathrm{~Hz}$ raised cosine. These envelope patterns can be thought of as coherent with the exception of additional modulation minima introduced in the "fast" pattern. Not surprisingly, a large CMR was obtained when the on-signal and flanking maskers were modulated by the same envelope pattern, either both fast or both slow. When the on-signal and flanking maskers had different patterns of modulation, the results depended on which masker(s) were modulated with the slower rate. The CMR was reduced by a factor of 2 or less when the flanking maskers had the slow pattern (and on-signal band had the fast pattern), but CMR was completely eliminated when the on-signal band had the slow pattern (and the flanking bands had the fast pattern). A similar pattern of results was obtained with ipsilateral and with contralateral presentation of flanking maskers, implicating central effects.

Whereas the results of Buss et al. (2009) are inconsistent with prior models of CMR based on across-frequency envelope comparison, they are consistent with the relative importance of spectral peaks as compared to dips in the profile analysis paradigm (Ellermeier, 1996; Lentz, 2005, 2006). For example, Ellermeier (1996) showed that under some conditions it is easier to detect an increment than a decrement to one tone in an otherwise equal-amplitude complex. Although the short-term power spectrum of a comodulated masker complex is flat as a function of frequency, decorrelating masker envelopes introduces peaks and/or dips in the dynamic spectral profile. If the on-signal band includes envelope minima not present in the flanking bands, the result would be a transient spectral dip at the signal frequency. On the other hand, if the flanking bands include envelope minima not present in the on-signal band, the result would be a transient spectral peak at the signal frequency. Spectral peaks at the signal frequency in the masker-alone stimulus could disrupt performance due to their salience and/or resemblance to spectral cues associated with an added tonal signal. The idea that transient spectral peaks play an important role in CMR is bolstered by the finding that masker modulation 
coherence does not improve sensitivity for signals associated with a transient reduction in stimulus level (Moore et al., 1990).

Comodulation masking release can be observed for a pure-tone signal across a wide range of signal frequencies (e.g., 250-4000 Hz; Grose and Hall, 1990; Haggard et al., 1990; Bacon et al., 2002). Some studies have shown a reduced effect at the high end of the audible spectrum (e.g., 6000-8000 Hz; Schooneveldt and Moore, 1987; Fantini et al., 1993), although Ernst and Verhey (2008) reported an increase in masking release between 4000 and $8000 \mathrm{~Hz}$. Some studies have also found reduced masking release at the low end of the spectrum (e.g., 250-500 Hz; Schooneveldt and Moore, 1987; Grose and Hall, 1990). The relative spectral position of the signal within the masker also has an effect on the size of CMR. Whereas masking release tends to be largest for a signal that is flanked on both sides by comodulated masker bands (Hall et al., 1988; Grose et al., 2005), there are conflicting data regarding the effects of spectral position of the signal when the masker is a pair of comodulated bands: in these cases CMR is often larger when the signal is centered in the high-frequency band than when it is centered in the low-frequency band (Schooneveldt and Moore, 1987; Hall et al., 1988; Cohen, 1991), but this is not always the case (Hall et al., 1984; McFadden, 1986; Cohen and Schubert, 1987). Interpretation of the published data on absolute and relative signal frequency effects in CMR for a tonal signal is further complicated by the finding of large individual differences and differential effects of within- and across-channel cues (e.g., Cohen and Schubert, 1987). It is also unknown whether frequency effects are similar for puretone and narrowband noise signals. Although published data are consistent with comparable effects of frequency for these two signal types, this conclusion is tempered by the limited range of signal frequencies $(500$ and $2000 \mathrm{~Hz}$; Hall and Grose, 1988) and the small number of observers $(n=3$; Fantini et al., 1993) tested.

The literature on profile analysis generates a clear expectation regarding sensitivity for spectral peak detection as a function of signal frequency. Green and his colleagues (Green and Mason, 1985; Bernstein and Green, 1988) reported a "bowl" pattern of signal thresholds as a function of component frequency for profile analysis stimuli. In one set of conditions, Bernstein and Green (1988) measured thresholds for detecting an increment to one tone in a set of 11 log-spaced standard tones at frequencies from 200 to 5000 $\mathrm{Hz}$, with level rove applied on an interval-by-interval basis. Thresholds varied from approximately $-11 \mathrm{~dB}$ re component level at $275 \mathrm{~Hz}$, to $-18 \mathrm{~dB}$ at $1000 \mathrm{~Hz}$, and $-12 \mathrm{~dB}$ at 3623 Hz. Similar patterns were found for different numbers of log-spaced standard tones. This regular relationship between signal threshold and frequency was described by the function

$$
y=20\left[\log (f / f \min )^{2}+b\right],
$$

where $y$ is the signal threshold in $\mathrm{dB}$ re component level, $f$ is the frequency of the signal in $\mathrm{Hz}, f \min$ is the frequency associated with the minimum threshold, and $b$ is a constant associated with overall sensitivity and the range of thresholds as a function of frequency. If CMR is based on a short- term spectral cue, then thresholds for analogous CMR stimuli should conform to the same function.

The present study assessed signal frequency effects using a traditional CMR stimulus, where the signal to be detected was a pure tone, and a hybrid stimulus, where the signal was a copy of the narrowband noise masker to which it was added (as in Hall and Grose, 1988). In both cases, comodulation of flanking and on-signal maskers was expected to result in a threshold reduction. The purpose of the present study was to test the hypothesis that thresholds plotted as a function of frequency would conform to a nonmonotonic, bowl pattern for both the pure-tone and the narrowband noise signals presented in a complex of coherently modulated masker bands. Such a result would support the idea that similar cues and auditory processes underlie performance in CMR and profile analysis. Conditions in which the flanking masker bands were presented contralateral to the signal and on-signal masker were included to allow discrimination of peripheral within-channel effects from more central across-channel effects. Different combinations of signal and masker frequencies were tested to assess relative and absolute frequency effects.

\section{GENERAL METHODS}

In all of the experiments, observers were normal-hearing adults, with pure-tone thresholds of $20 \mathrm{~dB}$ hearing level or less at octave frequencies from 250 to $8000 \mathrm{~Hz}$ (ANSI, 2004). None reported a history of chronic ear disease.

Stimuli were based on those of Bernstein and Green (1988). In one condition of that study, 11 stimulus components were equally spaced on a log scale, spanning the range 200 to $5000 \mathrm{~Hz}$, corresponding to a factor of approximately 1.38 between neighboring frequencies. These 11 frequencies correspond to the masker center frequencies in Experiment 1. Experiment 2 included an additional two frequencies above $5000 \mathrm{~Hz}$, but with the same factorial spacing. Experiments 3 and 4 used a subset of five or six neighboring masker frequencies. In all cases, maskers were played continuously over the course of a threshold estimation track and were composed of one or more 15-Hz-wide bands of Gaussian noise, each with a level of $50 \mathrm{~dB}$ sound pressure level (SPL).

The signal was a pure tone or a $15-\mathrm{Hz}$-wide band of noise centered on one of the masker frequencies. When the signal was a narrowband noise, it was an exact copy of the masker band to which it was added. The signal was ramped on and off using 20-ms raised-cosine ramps and had a total duration of $400 \mathrm{~ms}$. There were three masker conditions. In the on-signal condition the masker was a single band, centered on the signal frequency. In the all-coherent condition all of the masker bands shared a common pattern of amplitude modulation across frequency. In the all-random condition each band was a random, bandpass Gaussian noise.

Stimuli were generated in MATLAB prior to each threshold estimation track. For narrowband noise stimuli, bands were defined in the frequency domain by specifying the real and imaginary components within the pass-band using random draws from a normal distribution. In the all-coherent conditions each band was defined based on a single set of 
random draws, whereas in the all-random conditions all bands were based on independent draws. These arrays were transformed into the time domain using an inverse fast Fourier transform. With a $24.4 \mathrm{kHz}$ sampling rate, the $2^{18}$ point masker arrays repeated seamlessly every 10.7 s. Stimuli were played out via a digital-to-analog converter (RP2, TDT), routed through a headphone buffer (HB7, TDT) and presented over circumaural headphones (HD 265, Sennheiser). One exception was Experiment 2, for which deeply inserted earphones were used (ER-2, Etymotic). These transducers were used to achieve a nearly flat frequency response at the high stimulus frequencies unique to Experiment 2.

Stimuli were presented in a three-alternative forcedchoice, with 400-ms listening intervals and a 300-ms interstimulus interval. Each listening interval was marked with lights on a hand-held response box. Observers were instructed to select the interval in which the signal was presented, and feedback was provided visually after each response. Thresholds were estimated using a three-down, one-up procedure converging on the signal level associated with $79 \%$ correct (Levitt, 1971). For the tonal signal, the initial level adjustments were made in steps of $4 \mathrm{~dB}$. This was reduced to $2 \mathrm{~dB}$ after the second reversal. For the narrowband noise signal, initial level adjustments were made in steps of $2 \mathrm{~dB}$, reduced to $1 \mathrm{~dB}$ after the second reversal. ${ }^{1} \mathrm{~A}$ run continued until eight reversals had been obtained, and threshold was estimated as the mean signal level at the last six reversals. Three such estimates were obtained, with a fourth in cases where the first three spanned a range of $3 \mathrm{~dB}$ or more. The resulting three to four estimates were averaged. No practice was provided in these tasks.

Thresholds are reported and analyzed in units of $\mathrm{dB}$ SPL, the convention in the CMR literature. In a typical CMR experiment the relative starting phases of the signal and onsignal masker are random, as was the case for the tonal signal conditions in the present experiments. The narrowband noise signal and on-signal masker had the same starting phase, so addition of a fixed-level signal had a greater effect on the stimulus level for the narrowband noise than the tonal signal. The change in level associated with adding the signal to the on-signal masker $(\Delta L)$ is therefore included at the right axis in some figures.

For the purposes of evaluating the pattern of spectral effects, thresholds were expressed as the signal level minus the level of the on-signal masker ( $\mathrm{dB}$ re on-signal masker), following the conventions of the profile analysis literature. In a typical profile analysis experiment the starting phases of the signal and the standard are the same, as was the case for the narrowband noise signal in the present experiments. Thresholds for the tonal signal were therefore adjusted and represented as the level of a narrowband noise signal associated with the same $\Delta L{ }^{2}$

\section{EXPERIMENT 1}

The first experiment included stimulus conditions similar to those used by Bernstein and Green (1988) to demonstrate a bowl shape as a function of frequency in a profile analysis task. The task in that experiment was to detect an increment in level of one tone in a tonal complex, and the level of the complex was randomly roved on each presentation. In the present paradigm, the task was to detect a signal added to a complex of narrowband noise maskers. While the maskers were presented at a fixed average level, the inherent fluctuation of the narrowband noise samples could have disrupted the use of absolute level as a cue reflecting addition of the signal, analogous to the effect of stimulus level rove in the profile analysis paradigm. This possibility is consistent with reports that stimulus level fluctuation within a listening interval, such as that introduced by inherent fluctuation of a narrowband noise, impairs sensitivity to intensity increments (Bos and de Boer, 1966; Eddins, 2001).

The hypothesis of the first experiment was that thresholds as a function of frequency should follow the same bowl pattern as that demonstrated by Bernstein and Green (1988). The narrowband noise signals provide consistent spectral cues similar to those present in stationary profile analysis stimuli. If transient spectral cues are used to detect a puretone signal in a set of comodulated masker bands, then this bowl pattern should be evident in the all-coherent condition for the tonal signals, too. In addition to diotic test conditions, thresholds were obtained for dichotic presentation conditions, in which the signal and on-frequency masker were presented to one ear and flanking maskers to the other. If the pattern of thresholds as a function of frequency for diotic presentation were influenced by peripheral effects, then dichotic presentation should change that pattern. In contrast, if central effects are responsible for this pattern of diotic thresholds, then the pattern should be fundamentally similar for diotic and dichotic presentation.

\section{A. Methods \\ 1. Observers}

Observers were five adults, ranging in age from 23 to 46 years (mean of 31 years). All observers had previously completed at least $20 \mathrm{~h}$ of psychoacoustic listening unrelated to the current research, including CMR tasks.

\section{Stimuli}

There were 11 masker frequencies, equally spaced on a log scale and spanning 200-5000 Hz. Each masker was a $15-\mathrm{Hz}$-wide band of Gaussian noise presented at $50 \mathrm{~dB}$ SPL, played out continuously over the course of a track. The puretone or narrowband noise signal was at one of five even numbered masker frequencies: 276, 525, 1000, 1904, or $3624 \mathrm{~Hz}$. These stimuli were presented diotically, and thresholds were obtained in three masker conditions-on-signal, all-coherent, and all-random-for each of the five signal frequencies and two signal types, for a total of 30 conditions. In an additional manipulation, the on-signal masker and the signal were presented monaurally to the left ear and flanking maskers (when present) were presented to the right ear. These conditions are described as monaural/dichotic.

\section{Procedures}

All observers completed the diotic conditions prior to the monaural/dichotic conditions. Testing was blocked by 


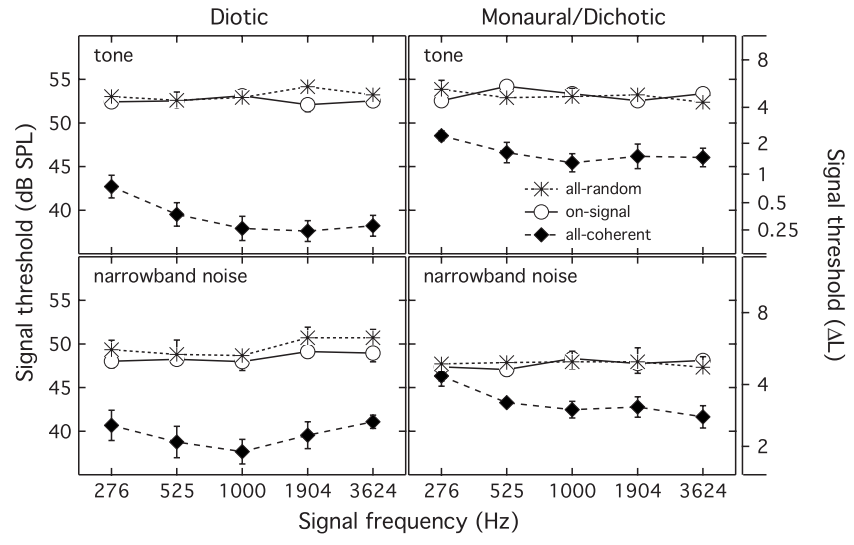

FIG. 2. Mean thresholds plotted as a function of signal frequency $(\mathrm{Hz})$. The left ordinate shows signal level in units of $\mathrm{dB}$ SPL, and the right ordinate shows the change in stimulus level for the $15-\mathrm{Hz}$-wide band at the signal frequency associated with addition of the signal, in units of $\Delta L$. Symbols reflect masker condition, as indicated in the legend. Error bars span \pm 1 standard error of the mean $(n=5)$.

signal frequency, with all three masker conditions for a given signal frequency completed in quasirandom order within that block. Blocks were completed in a different, quasirandom order by each observer. Thresholds in the on-signal condition were collected twice, once in the first half of the experiment (with diotic presentation) and again in the second half of the experiment (with monaural presentation).

\section{B. Results}

The pattern of thresholds was similar across the five observers, so mean data are reported. Figure 2 shows mean thresholds plotted in dB SPL as a function of signal frequency, with the associated values of $\Delta L$ shown at the right. Results are shown separately for diotic conditions and conditions in which the signal was monaural and flanking maskers were contralateral to the signal (left and right columns, respectively), and for tone and narrowband noise signals (top and bottom rows, respectively). Symbols reflect the masker condition, as indicated in the legend.

\section{Baseline conditions}

Thresholds for the on-signal masker conditions will be considered first. Thresholds in these conditions were relatively consistent across frequency, with means varying by 1-1.7 dB SPL. Results were also consistent across the onsignal masker conditions in the first and second stages of data collection (i.e., those associated with diotic and monaural presentation, respectively). For the pure-tone signal, mean thresholds were $52.5 \mathrm{~dB}$ (diotic) and $53.2 \mathrm{~dB}$ (monaural), and for the narrowband noise signal, mean thresholds were $48.5 \mathrm{~dB}$ (diotic) and $47.7 \mathrm{~dB}$ (monaural). A repeatedmeasures analysis of variance (ANOVA) was performed on these thresholds with two levels of EAR (diotic and monaural), two levels of SIGNAL (tone and narrowband noise), and five levels of FREQ $(276,525,1000,1904$, and $3624 \mathrm{~Hz})$. There was no effect of EAR $\left(F_{1.4}=0.01, p=0.93\right)$ and no effect of FREQ $\left(F_{4,16}=0.57, p=0.69\right)$, but there was a main effect of SIGNAL $\left(F_{1,4}=209.0, p<0.001\right)$. The signal level at threshold in the on-signal masker was $4.8 \mathrm{~dB}$ lower for the narrowband than for the tonal signal when thresholds are represented in $\mathrm{dB}$ SPL. This difference was smaller when thresholds were represented in units of $\Delta L$, with $0.4 \mathrm{~dB}$ lower thresholds for the tonal signal.

Thresholds in the all-random condition were similar to or slightly greater than those in the associated on-signal condition. For the diotic masker conditions, inclusion of random flanking bands elevated thresholds by $0.7 \mathrm{~dB}$ for the tonal signal and by $1.20 \mathrm{~dB}$ for the narrowband noise signal. For the dichotic masker conditions, mean thresholds in the onsignal and all-random conditions were within $0.1 \mathrm{~dB}$. A pair of repeated-measures ANOVA tests was performed to assess the significance of threshold elevation with inclusion of diotic, random flanking maskers, with separate tests for tonal and narrowband noise signal data. In both cases there were two levels of MASKER (on-signal and all-random) and five levels of FREQ $(276,525,1000,1904$, and $3624 \mathrm{~Hz})$. For the tonal signal there was a main effect of MASKER $\left(F_{1,4}\right.$ $=21.80, p<0.01)$, no effect of FREQ $\left(F_{4,16}=0.60, p\right.$ $=0.67)$, and no interaction $\left(F_{4,16}=2.52, p=0.08\right)$. For the narrowband noise signal, there was no effect of MASKER $\left(F_{1,4}=4.24, p=0.11\right)$, a significant effect of FREQ $\left(F_{4,16}\right.$ $=3.52, p<0.05)$, and no interaction $\left(F_{4,16}=0.48, p=0.75\right)$. Based on this result, the $1.2 \mathrm{~dB}$ mean threshold difference between all-random and on-signal thresholds should be treated with caution. Furthermore, the significant effect of signal frequency suggests that the $1.6 \mathrm{~dB}$ variation in mean threshold across frequency may reflect a small but reliable increase in threshold as a function of frequency, as suggested by the significant linear contrast associated with the main effect of frequency $\left(F_{1,4}=13.26, p<0.05\right)$.

\section{All-coherent masker}

In comparison to the two baseline conditions considered so far, thresholds in the all-coherent conditions were quite variable across frequency and across conditions. For the tonal signal, thresholds initially dropped with increasing frequency and then leveled off for both diotic and dichotic presentation. For the diotic stimulus conditions, thresholds dropped by $5 \mathrm{~dB}$ between 276 and $1904 \mathrm{~Hz}$. Calculating masking release as the difference in thresholds for allrandom and all-coherent conditions, a peak masking release of approximately $15 \mathrm{~dB}$ was obtained for the three highest signal frequencies. For the dichotic conditions, thresholds dropped by $3.1 \mathrm{~dB}$ between 276 and $1904 \mathrm{~Hz}$, and the peak masking release was approximately $7 \mathrm{~dB}$ at the three highest signal frequencies. Therefore, while there was reduced masking release with a monaural signal and contralateral flanking maskers, the variation of masking release with signal frequency for the tonal signal was broadly similar for the diotic and dichotic conditions.

In contrast to results obtained with a tonal signal, the pattern of thresholds for the narrowband noise signal conditions differed substantially for the diotic and dichotic stimulus conditions. For the diotic masker, thresholds initially fell with increasing frequency, dropping about $3.0 \mathrm{~dB}$ between 276 and $1000 \mathrm{~Hz}$. Above $1000 \mathrm{~Hz}$, thresholds rose with increasing frequency, with an increase of $3.4 \mathrm{~dB}$ between 1000 and $3624 \mathrm{~Hz}$. This pattern of results is similar to that ob- 


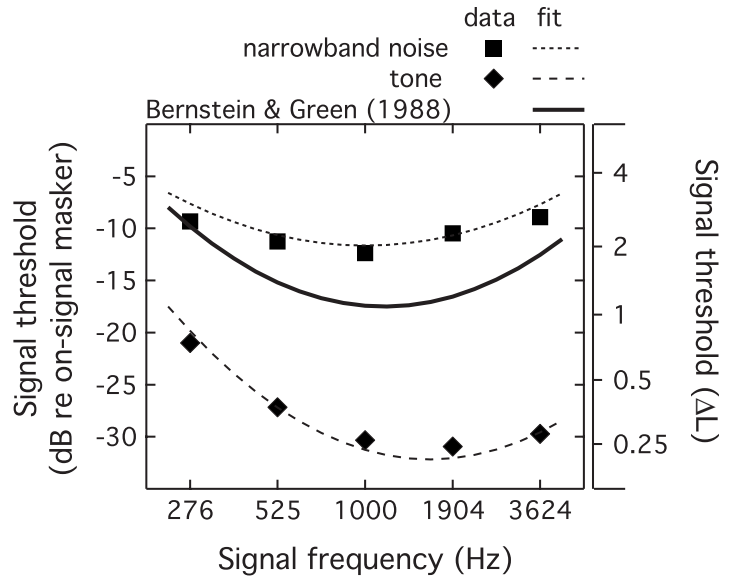

FIG. 3. Mean thresholds for the diotic all-coherent masker conditions are plotted in $\mathrm{dB}$ re on-signal masker, with pure-tone data represented as if the signal had been added in phase to the masker. The right ordinate shows the change in level, in units of $\Delta L$. Symbol shape reflects signal type, as defined in the legend. Lines show function fits, with the dark solid line indicating the fit to comparable profile analysis data of Bernstein and Green (1988).

served in published profile analysis data. Thresholds obtained with the monaural signal and dichotic maskers decreased consistently with increasing signal frequency, with no hint of an upturn in the function at the highest signal frequency.

A repeated-measures ANOVA was performed to assess the significance of the different pattern of thresholds obtained with the tonal and narrowband noise signals in diotic, all-coherent masker conditions. This analysis included two levels of SIGNAL (tone and narrowband noise) and five levels of FREQ $(276,525,1000,1904$, and $3624 \mathrm{~Hz})$. There was no effect of SIGNAL $\left(F_{1,4}=0.79, p=0.42\right)$, but there was a main effect of FREQ $\left(F_{4,16}=7.58, p<0.01\right)$, and there was a SIGNAL $\times$ FREQ interaction $\left(F_{4,16}=5.29, p\right.$ $<0.01)$. Repeating this analysis with the dichotic masker data, there was a main effect of SIGNAL $\left(F_{1,4}=21.14, p\right.$ $<0.05)$ and a main effect of FREQ $\left(F_{4,16}=8.26, p\right.$ $<0.001)$, but no SIGNAL $\times$ FREQ interaction $\left(F_{4,16}\right.$ $=0.72, p=0.59)$. This result is consistent with the visual impression that in the all-coherent conditions, the pattern of thresholds as a function of frequency differed for the two signal types for diotic, but not dichotic, masker conditions.

\section{Fits to diotic, all-coherent data}

Functions were fitted to the data to characterize the different patterns of results for the two signal types in the allcoherent masker conditions. To facilitate comparison with the previous profile analysis data of Bernstein and Green (1988), thresholds were represented as $\mathrm{dB}$ re on-signal masker. Pure-tone thresholds were adjusted to the level of a narrowband noise signal associated with the same $\Delta L$ as that tone. Mean data for the diotic, all-coherent conditions are plotted in Fig. 3, with the associated fits. The narrowband noise signal thresholds are shown as squares, and tonal signal thresholds are shown as diamonds.

Initial fits of Eq. (1) to the narrowband noise signal thresholds were very poor, probably because the slope of the threshold as a function of frequency was relatively shallow. This was addressed by introducing a scalar, $a$, to Eq. (1), as follows:

$$
y=a \times 20\left[\log (f / f \min )^{2}+b\right] .
$$

Not surprisingly, the resulting three-parameter fit was more consistent with the pattern of mean data $\left(R^{2}=0.93\right)$. All subsequent fits were made using Eq. (2) and individual data, normalized to account for small individual differences in overall sensitivity (on the order of $\pm 2.5 \mathrm{~dB}$ ). Two fits were performed on each set of data, one in which all three parameters were free to vary ( $\min , a$, and $b$ ), and one in which fmin was fixed at $1150 \mathrm{~Hz}$, the minimum reported by Bernstein and Green (1988) for tonal profile analysis stimuli.

For the narrowband noise data in the diotic all-coherent conditions, the best-fitting fmin was $1004 \mathrm{~Hz}$ for the threeparameter fit $\left(R^{2}=0.55\right)$. This fit was not significantly better than a two-parameter fit fixing $f \min$ at $1150 \mathrm{~Hz}\left(R^{2}\right.$ $\left.=0.49 ; \quad F_{1,22}=3.06, p=0.09\right)$. Repeating this procedure for the tonal signal data resulted in a three-parameter fit with fmin of $1613 \mathrm{~Hz}\left(R^{2}=0.80\right)$. This fit was significantly better than a two-parameter fit with fmin of $1150 \mathrm{~Hz}\left(R^{2}\right.$ $\left.=0.58 ; F_{1,22}=24.51, p<0.0001\right)$. Figure 3 shows the three-parameter fits to individual data [Eq. (2)], with the fit to mean data reported by Bernstein and Green (1988) for tonal profile analysis stimuli [Eq. (1)] indicated with the heavy solid line. Comparing the two functions associated with the present data set, both generally conform to a bowl shape, with a reduced masking release and a lower value of fmin for the narrowband noise than for the pure-tone signal. One caveat to this summary is that the tonal data provide very weak evidence of an increase in pure-tone threshold at the high-frequency end of the spectrum, with an increase in threshold between 1000 and $3624 \mathrm{~Hz}$ of only $0.3 \mathrm{~dB}$.

\section{Fits to dichotic, all-coherent data}

Equation (2) was also fitted to individual data for the dichotic all-coherent conditions. For the narrowband noise data in the dichotic all-coherent conditions, the best-fitting fmin was $2456 \mathrm{~Hz}$ for the three-parameter fit $\left(R^{2}=0.55\right)$. This fit was significantly better than a two-parameter fit fixing $f \min$ at $1150 \mathrm{~Hz}\left(R^{2}=0.25 ; F_{1,22}=14.13, p<0.005\right)$. Repeating this procedure with the data for the tonal signal resulted in a three-parameter fit with fmin of $1555 \mathrm{~Hz}\left(R^{2}\right.$ $=0.39$ ). In this case the three-parameter fit was not significantly better than a two-parameter fit, with fmin of $1150 \mathrm{~Hz}$ $\left(R^{2}=0.30 ; F_{1,22}=3.37, p=0.08\right)$. This result could be due in part to the relatively poor fit achieved in the threeparameter case in combination with the smaller effect of signal frequency in dichotic, as compared to diotic, conditions. As with the diotic tonal data fits, interpretation of these estimates of fmin is tentative given the lack of evidence of threshold elevation at high frequencies.

\section{Discussion}

The most interesting aspect of the data from Experiment 1 is the pattern of thresholds for the all-coherent conditions as a function of frequency. Thresholds for the narrowband 
noise signal in the diotic all-coherent masker condition resembled the bowl pattern demonstrated for profile analysis, with a minimum at $1004 \mathrm{~Hz}$. This was not significantly different from the $1150 \mathrm{~Hz}$ minimum reported by Bernstein and Green (1988). In contrast, tonal thresholds in the diotic allcoherent conditions fell as a function of frequency, with a minimum estimated at $1613 \mathrm{~Hz}$, significantly higher than $1150 \mathrm{~Hz}$. Fits to data for the dichotic conditions also resulted in mean estimates of fmin above $1150 \mathrm{~Hz}$, although this difference was only significant for the narrowband noise signal. These results provide some preliminary support for the conclusion that thresholds in the diotic all-coherent conditions conform to a bowl pattern, albeit with a higher minimum for tonal than for narrowband noise signals. This conclusion is tempered by three considerations. First is the relatively small range of thresholds in the narrowband noise signal conditions, with means spanning only $3 \mathrm{~dB}$; this effect is small compared to that observed with the profile analysis stimulus, where thresholds spanned a range of about $8 \mathrm{~dB}$ over a comparable range of signal frequencies. Second is the lack of evidence of an up-turn in the function associated with the tonal signal. Although the fitted function is consistent with a minimum between the two highest signal frequencies tested, the mean thresholds measured at these two points differed by less than $1 \mathrm{~dB}$. Third is the relatively poor data fit of Eq. (2) in some conditions, indicating that the "bowl" shape may not provide a good descriptor of the data pattern.

In contrast to the "bowl" pattern observed with narrowband noise signals and diotic maskers, thresholds tended to fall nearly monotonically with increasing frequency for dichotic masker presentation for both the tonal and the narrowband noise signals. This result introduces the possibility that within-channel effects could be responsible for the increase in threshold at the higher signal frequencies with the narrowband noise signal and diotic masker. This possibility is discussed in Sec. VII. An additional consideration in the interpretation of the diotic/dichotic masker differences in the present data set is that the signal and on-signal masker were presented to both ears in the diotic condition, whereas the signal and on-signal band were monaural in the dichotic conditions. While this difference would not be expected to affect the pattern of thresholds as a function of signal frequency, possible consequences of this difference were assessed empirically in Experiment 2.

\section{EXPERIMENT 2}

Experiment 1 was repeated with a spectrally wider stimulus that included two additional maskers bands and allowed threshold estimation above $3624 \mathrm{~Hz}$. The goal of this manipulation was to further assess the hypothesis that thresholds for the tonal signal conform to a nonmonotonic bowl pattern, albeit with a relatively high-frequency minimum compared to that for narrowband noise signals. Secondary goals of this experiment were to replicate the bowl pattern with the narrowband noise signal and to assess the possible effects of diotic, as compared to monaural, presentation of stimuli in the all-coherent masker conditions.

\section{A. Methods \\ 1. Observers}

Observers were six adults, ranging in age from 18 to 53 (mean of 27 years). One observer had previously participated in Experiment 1. The other five had prior experience in psychoacoustic tasks unrelated to the present research, including CMR tasks.

\section{Stimuli}

Stimuli were similar to those used in Experiment 1, but included two additional high-frequency masker bands and one additional high-frequency signal. The masker was made up of bands centered on each of 13 frequencies, equally spaced on a log scale and spanning the range 200 to 9522 Hz. Signals were centered on 276, 525, 1000, 1904, 3624, or $6899 \mathrm{~Hz}$. In the ipsilateral masker conditions, the signal and all masker bands were presented to the right ear. In the contralateral masker conditions, the signal and on-signal masker were presented to the right ear, and the flanking maskers were presented to the left ear. Additional conditions included diotic presentation of both the signal and the maskers. Thresholds were collected at all signal frequencies for the all-coherent condition, whereas thresholds in the on-signal and all-random conditions were collected only for ipsilateral masker conditions and only at 1000 and $6899 \mathrm{~Hz}$.

\section{Procedures}

In contrast to other the experiments reported here, stimuli were presented using deeply inserted earphones (ER-2, Etymotic), a change made to ensure a nearly flat frequency response for the extended stimulus frequency range used in this experiment. As in the previous experiment, thresholds were blocked by condition, with all conditions at a given signal frequency performed in random order. The one exception was that thresholds for the diotic all-coherent conditions were obtained prior to those in any other conditions.

\section{B. Results}

Mean results are plotted as a function of signal frequency in Fig. 4. Results for the tonal signal appear in the top panel, and those for the narrowband noise signal in the bottom panel.

\section{Baseline conditions}

As for the previous data, performance in the on-signal and all-random conditions was similar, with mean thresholds of 50.7 and $51.9 \mathrm{~dB}$ SPL, respectively. A repeated-measures ANOVA was performed on thresholds in the eight baseline conditions, with two levels of SIGNAL (tone and narrowband noise), two levels of MASKER (on-signal and allrandom), and two levels of FREQ (1000 and $6899 \mathrm{~Hz})$. This analysis indicated main effects of SIGNAL $\left(F_{1.5}=129.1, p\right.$ $<0.0001)$, FREQ $\left(F_{1.5}=7.24, p<0.05\right)$, and MASKER $\left(F_{1.5}=6.71, p<0.05\right)$. None of the interactions reached significance $(p \geq 0.1)$. On average thresholds were $0.7 \mathrm{~dB}$ lower 


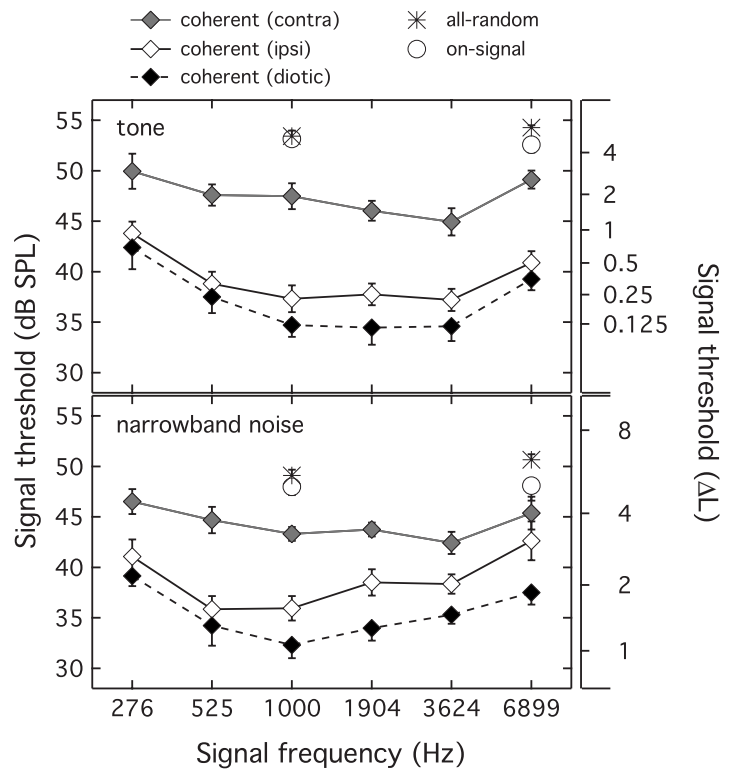

FIG. 4. Mean thresholds in dB SPL plotted as a function of signal frequency $(\mathrm{Hz})$ for the extended masker range. The right ordinate shows the change in level, in units of $\Delta L$. Symbols reflect masker condition, as indicated in the legend. Error bars span \pm 1 standard error of the mean $(n=6)$.

at 1000 than $6899 \mathrm{~Hz}, 1.2 \mathrm{~dB}$ lower for on-signal than random masker conditions, and $4.4 \mathrm{~dB}$ lower for the narrowband noise than the tonal signal.

\section{Diotic and ipsilateral, all-coherent}

Thresholds at the first five signal frequencies in the diotic, all-coherent conditions followed the same pattern observed in Experiment 1 (compare black diamonds in Fig. 4. and the left panel of Fig. 2). Thresholds for the narrowband noise signal decreased as frequency increased up to about $1000 \mathrm{~Hz}$ and then rose. Pure-tone thresholds dropped between 276 and $1000 \mathrm{~Hz}$, and were nearly constant between 1000 and $3624 \mathrm{~Hz}$. At the highest frequency of $6899 \mathrm{~Hz}$, thresholds for the tonal signal rose. A similar pattern was observed with the ipsilateral masker. Thresholds in the ipsilateral, all-coherent conditions were on average $2.7 \mathrm{~dB}$ higher than those in the associated diotic conditions. A repeated-measures ANOVA was performed to confirm this difference, with two levels of PRESENTATION (ipsilateral and diotic), two levels of SIGNAL (tone and narrowband noise), and six levels of FREQ (256-6899 Hz). All three main effects were significant, including SIGNAL $\left(F_{1,5}\right.$ $=11.10, p<0.05)$, FREQ $\left(F_{5,25}=16.03, p<0.0001\right)$, and PRESENTATION $\left(F_{1,5}=65.74, p<0.001\right)$. The interaction between SIGNAL and FREQ was significant $\left(F_{5,25}\right.$ $=4.34, p<0.01)$, but no other interactions reached significance $(p>0.05)$. These results confirm that the pattern of all-coherent thresholds differs for the two signal types, but not for diotic as compared to ipsilateral (monaural) presentation.

To facilitate comparison of thresholds for the diotic, allcoherent conditions in Experiments 1 and 2, Eq. (2) was fitted to the thresholds between 276 and $3624 \mathrm{~Hz}$, the frequencies common to both experiments. For the tonal signal, this resulted in an fmin estimate of $1947 \mathrm{~Hz}\left(R^{2}=0.66\right)$ for
Experiment 2, compared to $1613 \mathrm{~Hz}$ for Experiment 1. Holding fmin constant at $1613 \mathrm{~Hz}$ and refitting the data with two free parameters resulted in a nonsignificant reduction in percent of variance accounted for $\left(R^{2}=0.64 ; F_{1,27}=1.26, p\right.$ $=0.27$ ). A three-parameter fit to the data for the narrowband noise signal of Experiment 2 resulted in an fmin estimate of $1245 \mathrm{~Hz}\left(R^{2}=0.67\right)$, compared with $1004 \mathrm{~Hz}$ for Experiment 1. Holding fmin constant at $1004 \mathrm{~Hz}$ and refitting the data reduced the percent of variance accounted for significantly $\left(R^{2}=0.51 ; \quad F_{1,27}=13.73, p<0.005\right)$. Overall thresholds were lower in the diotic conditions for Experiment 2 than for Experiment 1. Averaged across the signal frequencies common to both experiments, this difference was $2.5 \mathrm{~dB}$ for the tonal signal and $4.8 \mathrm{~dB}$ for the narrowband noise signal. Although the general pattern of results was similar across experiments, with a lower value of $f$ min for the narrowband than for the tonal signal, there were some differences, such as the lower fmin associated with the narrowband noise signal in Experiment 1 than in Experiment 2. Different results could be due to individual variability or to the wider range of masker frequencies in the second experiment.

\section{Contralateral, all-coherent}

As in the ipsilateral, all-coherent conditions with the tonal signal, thresholds in the contralateral, all-coherent conditions for both signal types fell with increasing signal frequency up to $3624 \mathrm{~Hz}$, but then rose at the highest signal frequency of $6899 \mathrm{~Hz}$. A repeated-measures ANOVA was performed to compare the frequency effects for the two signal types. There were two levels of SIGNAL (tonal and narrowband noise) and six levels of FREQ (256-6899 Hz). Both main effects were significant, including SIGNAL $\left(F_{1,5}=44.00, p<0.001\right) \quad$ and FREQ $\quad\left(F_{5,25}=5.87, p\right.$ $<0.001)$, but the interaction was not significant $\left(F_{5,25}\right.$ $=0.52, p=0.76)$. Whereas signal type resulted in different signal frequency effects in diotic and ipsilateral conditions, it did not play a role in the threshold-by-frequency pattern of contralateral data.

\section{Discussion}

Tonal thresholds rose at the high end of the masker spectrum when the range of masker frequencies was extended above $5000 \mathrm{~Hz}$. In both Experiments 1 and 2, thresholds for the all-coherent, tonal signal conditions were fitted significantly better by a function with a minimum near 1600-1900 $\mathrm{Hz}$ than $1150 \mathrm{~Hz}$. This finding is consistent with the conclusion that a bowl pattern characterizes thresholds as a function of frequency for both the tonal and narrowband noise signal types, but that the minimum of that bowl is shifted to higher frequencies for the tonal than for the narrowband noise signal. The pattern of thresholds in contralateral masker conditions with both signal types resembled that obtained with the tonal signal and ipsilateral masker; that is, thresholds fell gradually with increasing frequency and then increased at the highest signal frequency tested. In contrast, thresholds with the ipsilateral maskers and narrowband noise signal had a minimum near $1000 \mathrm{~Hz}$. The pattern of thresholds was similar for ipsilateral (monaural) and diotic masker presentation, 
although thresholds in the diotic conditions were slightly lower. This diotic advantage has been noted in other stimulus conditions thought to be limited by internal rather than by external noise (Langhans and Kohlrausch, 1992).

\section{EXPERIMENT 3}

The origin of the bowl data pattern in profile analysis is unknown, but it has been shown that the minimum of the bowl is affected by both absolute frequency and relative frequency of the signal within the masker complex (Green and Mason, 1985; Green et al., 1987). The absolute frequency effect in profile analysis leads to the prediction that if thresholds in the comodulation condition of the CMR paradigm are based on short-term spectral cues similar to those underlying profile analysis, then those thresholds should also differ across frequency. There is evidence of a frequency effect for a pure-tone signal in a comodulated masker (e.g., Schooneveldt and Moore, 1987), but little is known about the effect of frequency for a narrowband noise signal or the relation of this finding to the use of dynamic spectral cues.

\section{A. Methods}

\section{Observers}

Observers were nine adults, ranging from 23 to 51 years (mean of 30 years). All observers had previously completed at least $8 \mathrm{~h}$ of psychoacoustic listening unrelated to the current research. An additional observer with no prior listening experience was also recruited, but her data were omitted due to poor reliability and high thresholds. Replicate thresholds for this observer spanned a range of $15 \mathrm{~dB}$ in one condition, more than twice the range obtained in any condition for the other observers, and her thresholds were on average $9 \mathrm{~dB}$ above the group mean.

\section{Stimuli}

Stimuli were based on the diotic, all-coherent conditions of Experiment 1. In contrast to that experiment, a subset of five neighboring masker bands was presented in each condition. Those maskers were the on-signal band and the two neighboring bands above and below the signal frequency. For example, when the signal frequency was $381 \mathrm{~Hz}$, the masker consisted of the first five bands (200, 276, 381, 525, and $725 \mathrm{~Hz}$ ). Three signal frequencies were used: 381,1000 , and $2627 \mathrm{~Hz}$.

\section{Procedures}

The threshold estimation procedure was identical to that used in Experiment 1. Conditions were completed in blocks by condition, with the order of conditions randomized across observers.

\section{B. Results}

Figure 5 shows thresholds plotted as a function of signal frequency, with results for the tonal signal in the top panel and those for the narrowband noise signal in the bottom panel. Open symbols indicate individual observers' data, and the connected black diamonds show the mean across observ-

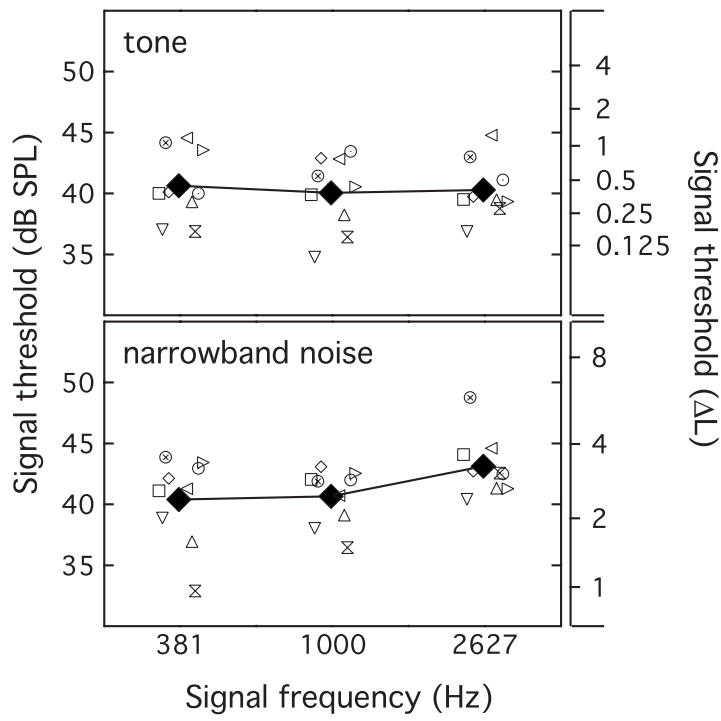

FIG. 5. Thresholds in dB SPL for the restricted-frequency conditions of Experiment 3, plotted as a function of signal frequency. The right ordinate shows the change in level, in units of $\Delta L$. The top panel shows results for the pure-tone signal, and the bottom panel shows results for the narrowband noise signal. Individuals' thresholds are shown by small symbols, and filled diamonds connected with lines shows the mean.

ers. While there were marked individual differences, the mean thresholds were nearly constant across frequency for the tonal signal, ranging from 40.0 to $40.6 \mathrm{~dB}$ SPL. Mean thresholds for the narrowband noise signal were approximately $40.5 \mathrm{~dB}$ for the lower two signal frequencies, but rose to $43.1 \mathrm{~dB}$ at the highest signal frequency. These thresholds were not as low as the lowest threshold measured in comparable conditions of Experiment 1, with values of $37.6 \mathrm{~dB}$ $(1904 \mathrm{~Hz})$ for the tonal signal and $37.6 \mathrm{~dB}(1000 \mathrm{~Hz})$ for the narrowband noise signal. Thresholds in the present conditions were more comparable to those measured for a signal in the fourth masker band (at $525 \mathrm{~Hz}$ ) in Experiment 1, with means of 39.5 and $38.7 \mathrm{~dB}$, respectively.

A repeated-measures ANOVA was performed to assess the significance of absolute frequency effects in these thresholds. There were two levels of signal TYPE (tone and narrowband noise) and three levels of signal FREQ (381, 1000, and $2627 \mathrm{~Hz}$ ). The main effect of TYPE failed to reach significance $\left(F_{1,8}=4.65, p=0.06\right), \quad$ as did FREQ $\left(F_{2,16}\right.$ $=2.24, p=0.14)$. The interaction, however, was significant $\left(F_{2,16}=8.02, p<0.01\right)$. A contrast indicated a significant interaction with TYPE for 1000 and $2624 \mathrm{~Hz}\left(F_{1,8}\right.$ $=15.72, p<0.001)$ but not for 381 and $1000 \mathrm{~Hz}\left(F_{1,8}\right.$ $=0.95, p=0.36)$. These results are consistent with an absolute frequency effect for the narrowband noise but not for the pure-tone signal. However, this effect of frequency was small, on the order of $2.5 \mathrm{~dB}$, and subject to large individual differences.

\section{Discussion}

Stimuli in Experiment 3 included five masker bands, all coherently modulated and separated in frequency by a factor of approximately 1.38 . The added signal was spectrally centered in the masker complex, so results should be dominated 
by absolute frequency effects (in contrast to relative frequency effects). Thresholds for the narrowband noise signal rose slightly when the signal frequency rose from 1000 to $2627 \mathrm{~Hz}$. This result is similar to the results of Green and Mason (1985), in which the signal was an increment in level of the central component of a five-tone complex, with components separated in frequency by a factor of 1.17 . In that study, mean threshold rose with increasing signal frequency between 552 and $3808 \mathrm{~Hz}$. The increase in narrowband signal thresholds between 1000 and $2627 \mathrm{~Hz}$ in the present study is consistent with the idea that absolute frequency effects contributed to the rising, high-frequency portion of the bowl threshold pattern observed in the narrowband signal, all-coherent diotic masker conditions of Experiment 1.

While thresholds for the narrowband noise signal rose at the highest signal frequency, thresholds for the tonal signal were relatively constant as a function of frequency. The fact that absolute frequency effects were observed with the narrowband noise but not the tonal signal in the present experiment is also consistent with the results of Experiment 1. Based on the three-parameter fits to data in the diotic allcoherent conditions of Experiment 1, narrowband noise thresholds would be expected to rise by $2.2 \mathrm{~dB}$ between 1000 and $2627 \mathrm{~Hz}$. Tonal thresholds would be expected to remain nearly constant over that range, with a predicted increase of less than $0.1 \mathrm{~dB}$. In this context, a frequency effect that is limited to the narrowband noise signal conditions of the present experiment could reflect the same processes that are responsible for the high-frequency thresholds observed in Experiment 1.

\section{EXPERIMENT 4}

Although an absolute frequency effect was found in Experiment 3 , this effect was more consistent with threshold elevation at the high-frequency end of the masker complex than threshold elevation at the low-frequency end. For the narrowband noise signal, thresholds were higher at 2627 than $1000 \mathrm{~Hz}$, indicating that absolute frequency effects could play a role in the relatively poor thresholds at high frequencies for the narrowband noise signal in Experiment 1. However, thresholds at the two lower frequencies of Experiment 3 were comparable, indicating that absolute frequency fails to account for relatively poor thresholds below $1000 \mathrm{~Hz}$ in Experiment 1. Because absolute frequency effects are inconsistent with poor thresholds below $1000 \mathrm{~Hz}$, the final experiment evaluated the possibility that relative frequency might also play a role in the magnitude of masking release as a function of frequency. Of particular interest was the possible influence of relative frequency effects on the low-frequency portion of the bowl pattern of thresholds, where thresholds fall with increasing signal position in the masker spectrum.

\section{A. Methods}

\section{Observers}

Observers were seven adults, ranging in age from 21 to 53 years (mean of 30 years). All observers had previously completed at least $5 \mathrm{~h}$ of psychoacoustic listening unrelated to the current research, including studies of CMR.

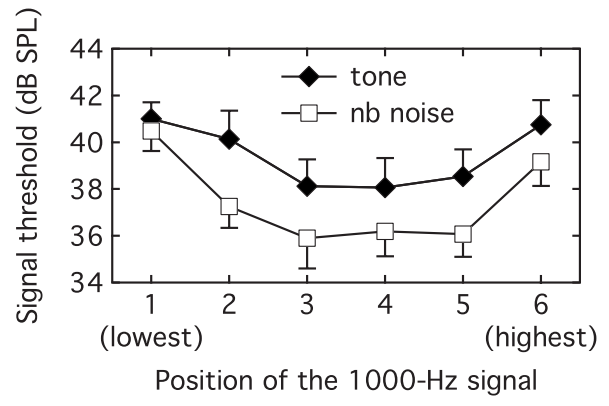

FIG. 6. Thresholds in the restricted-frequency conditions of Experiment 4 plotted as a function of the relative position of the $1000 \mathrm{~Hz}$ on-signal band within the six-band comodulated masker complex. Symbols reflect the signal condition, as indicated in the legend. Error bars span \pm 1 standard error of the mean $(n=7)$.

\section{Stimuli}

Stimuli were based on the diotic, all-coherent conditions of Experiment 1. A subset of six neighboring masker bands was presented in each condition, one of which was the band centered on $1000 \mathrm{~Hz}$. The signal frequency was $1000 \mathrm{~Hz}$ in all conditions. Stimulus conditions varied in terms of the flanking masker band frequencies, and these conditions will be referred to with the ordinal position of the on-signal band in frequency (low to high). When the signal was in band 1, the on-signal band was positioned at the low-frequency edge of the masker complex and the flanking maskers were centered on 1380, 1904, 2627, 3624, and $5000 \mathrm{~Hz}$. In contrast, when the signal was in band 6, the on-signal band was at the high-frequency edge of the complex and the flanking maskers were centered on 200, 276, 381, 525, and $725 \mathrm{~Hz}$. Due to a programming error, the level of each masker band was $49 \mathrm{~dB}$ SPL rather than $50 \mathrm{~dB}$ SPL (the masker level used in previous experiments).

\section{Procedures}

Testing was blocked by signal position, with the order randomized for each observer. For each signal position, testing began with either the tonal or the narrowband noise signal, selected at random. Testing continued in this manner through the remaining five signal positions, with an independent randomization of signal type at each position.

\section{B. Results and discussion}

Mean thresholds are plotted in Fig. 6 as a function of on-signal band position, with symbols indicating signal type, as shown in the legend. Recall that the signal was always centered on $1000 \mathrm{~Hz}$, and the abscissa indicates its relative position within the six-band masker complex. As in Experiments 1 and 2, thresholds varied nonmonotonically with relative signal frequency, with lowest thresholds in the middle positions.

For both the tonal and narrowband noise signals, thresholds initially fell as a function of position of the $1000 \mathrm{~Hz}$ signal, dropping between bands 1 and 3. Thresholds then leveled off, rising again when the on-signal band was at the high-frequency edge of the masker complex. This impression was confirmed with a repeated-measures ANOVA, with two 
levels of SIGNAL (tone and narrowband noise) and six levels of relative signal POSITION (bands 1-6). This analysis resulted in main effects of SIGNAL $\left(F_{1,6}=10.51, p\right.$ $<0.05)$ and POSITION $\left(F_{5,30}=18.27, p<0.001\right)$, but no interaction $\left(F_{5,30}=1.22, p=0.32\right)$. A repeated contrast indicated a significant difference between thresholds for bands 1 and $2 \quad\left(F_{1,6}=10.56, p<0.05\right)$, bands 2 and $3\left(F_{1,6}\right.$ $=21.92, p<0.01)$, and bands 5 and $6\left(F_{1,6}=48.22, p\right.$ $<0.001)$. Thresholds for the other two sequential pairs of bands were not significantly different $(p<0.70)$.

In the present conditions, the lowest and highest relative signal positions were associated with the spectral edge of the masker complex. In contrast, in the previous experiments there was always at least one masker band above and below the signal frequency. Therefore, results for signal positions from bands 2 to 5 may be most pertinent to interpretation of the nonmonotonic frequency effects demonstrated in Experiments 1 and 2. Excluding the two edge positions (bands 1 and 6), the only evidence of a relative frequency effect is the difference between bands 2 and 3, a mean effect of $1.7 \mathrm{~dB}$. In other words, having two comodulated maskers below the signal results in better performance than having just one, but there is no analogous effect at the high-frequency end of the threshold function. Provided that the signal is not at the spectral edge of the complex, relative frequency effects are small and evident only in the low-frequency portion of the bowlshaped function.

\section{GENERAL DISCUSSION}

It was predicted that the pattern of thresholds in the presence of a coherently modulated masker complex would resemble the bowl observed in profile analysis based on the hypothesis that CMR depends on dynamic spectral cues similar to those underlying performance in profile analysis. To that end, stimuli were closely modeled after those of Bernstein and Green (1988), but using 15-Hz-wide bands of noise instead of tonal pedestals. The signal to be detected was either a pure tone centered on one of these bands or a scaled copy of a single masker band. Conditions with the narrowband noise signal could be described as dynamic profile analysis stimuli (e.g., Green and Nguyen, 1988), whereas those with the tonal signal was more typical of CMR stimuli.

\section{A. Summary of results}

Experiments 1 and 2 showed that while thresholds in the on-signal and random masker conditions were relatively constant across frequency, mean thresholds in conditions with comodulated masker bands differed by up to $7.9 \mathrm{~dB}$ as a function of frequency. The pattern of masking release as a function of signal frequency depended on the signal type and whether flanking maskers were presented ipsilateral or contralateral to the signal and on-signal masker band. When the signal was a copy of the on-signal masker band, thresholds in the diotic all-coherent conditions formed a nonmonotonic bowl pattern with a minimum near $1000 \mathrm{~Hz}$, similar to that observed in the profile analysis literature (Green, 1988). With a tonal signal, thresholds in analogous masker conditions improved with increasing frequency up to approximately 1600$1900 \mathrm{~Hz}$, beyond which thresholds rose. These two patterns of results were found for both monaural and diotic stimulus presentation, with some indication of better performance in the latter condition. The finding of a nonmonotonic bowl pattern in all-coherent masker conditions lends some credibility to the idea that dynamic spectral cues underlie the detection advantage associated with coherent masker modulation, with the caveat that the threshold minimum occurred at higher frequencies for the tonal than for the narrowband noise signal. If the bowl pattern observed in the CMR conditions of the present study reflects the use of dynamic spectral cues, then the finding of a different frequency minimum for the two signal types may reflect a difference in the way the associated cues are encoded or used.

Thresholds in the contralateral, all-coherent conditions fell with increasing frequency, except for an increase at 6899 $\mathrm{Hz}$, the highest signal frequency tested in Experiment 2. In contrast to the diotic and ipsilateral data, the pattern of thresholds with contralateral flanking masker presentation did not appear to differ for the tonal and narrowband noise signal types. The nonmonotonicity of contralateral data is consistent with the conclusion that the bowl pattern of thresholds is not due solely to peripheral effects, but the differences between ipsilateral and contralateral data indicate that the pattern of thresholds as a function of frequency could be affected by peripheral processes.

Green and Mason (1985) argued that the bowl pattern formed by thresholds in the profile analysis paradigm is affected by both the absolute frequency of the signal and the relative spectral position of the signal in the masker complex. Analogous effects were observed in the pattern of allcoherent thresholds of Experiments 3 and 4. When the signal was centered in a five-band masker, thresholds for the narrowband noise signal were higher at $2627 \mathrm{~Hz}$ than at 381 or $1000 \mathrm{~Hz}$. Thresholds for the tonal signal, however, were comparable across these three frequencies. This result is consistent with the interpretation that at least part of the highfrequency portion of the bowl is due to absolute frequency effects and that this high-frequency effect occurs at lower frequencies for the narrowband noise than for the tonal signal. In contrast, Experiment 4 showed evidence of a relative frequency effect: a nonmonotonic bowl shape was obtained for a $1000 \mathrm{~Hz}$ signal as a function of its relative position within the six-masker complex, a stimulus formed by including masker bands above and/or below the $1000 \mathrm{~Hz}$ on-signal band. Excluding conditions in which the signal was at the spectral edge of the stimulus, relative frequency effects were evident only for a signal near the low end of the masker spectrum. Data from Experiments 3 and 4 are consistent with the hypothesis that relative frequency effects influence the low-frequency portion of the bowl, and absolute frequency effects play a larger role in the high-frequency portion. There is some precedent for the idea that relative and absolute frequency effects dominate performance at low and high frequencies, respectively. In a study of profile analysis with stationary stimuli, Green et al. (1987) argued that relative 
effects dominate the pattern of thresholds at low frequencies, with absolute frequency effects taking on more importance for stimuli extending above $5000 \mathrm{~Hz}$.

Although the frequency effects found in Experiments 3 and 4 were modest compared to those in Experiments 1 and 2 , the first two experiments included more flanking masker bands (with 10-12 flanking bands) than the latter two experiments (with 4-5 flanking bands). Since the magnitude of CMR increases with increasing number of bands (e.g., Hall et al., 1990), the inclusion of more comodulated masker bands in the first two experiments could have increased the magnitude of absolute and/or relative frequency effects.

\section{B. Frequency effects in relation to CMR literature}

Several previous reports on CMR with a pure-tone signal and narrowband maskers are consistent with the present findings of an increase in masking release with increasing signal frequency between 250 and $4000 \mathrm{~Hz}$, and a reversal of this trend between 4000 and $8000 \mathrm{~Hz}$ (Cohen and Schubert, 1987; Schooneveldt and Moore, 1987; 1989; Fantini et al., 1993). In several cases, these trends have been attributed to within-channel effects (Cohen and Schubert, 1987; Schooneveldt and Moore, 1989). One study, in particular, by Grose and Hall (1990) reported thresholds for conditions similar to those of the present experiment. In that study, the masker was a set of nine bands of noise, each $20 \mathrm{~Hz}$ wide and logarithmically distributed between 276 and $3624 \mathrm{~Hz}$. The signal was a pure tone at one of those center frequencies, omitting the highest and lowest frequencies. The purpose of that study was to document the effects of signal frequency uncertainty, rather than signal frequency effects, but the data indicate a decrease in thresholds of about $4 \mathrm{~dB}$ between signal frequencies of 381 and $725 \mathrm{~Hz}$, and relatively constant thresholds between 1000 and $2627 \mathrm{~Hz}$.

There is some precedent in the CMR literature for the finding of comparable frequency effects with ipsilateral and contralateral maskers. Hall et al. (1990) measured detection thresholds for a $700 \mathrm{~Hz}$ pure tone added to a 20-Hz-wide band of noise centered on the signal frequency in the presence of one or more comodulated flanking masker bands. Stimuli were presented either diotically or dichotically, with the signal and on-signal masker band to one ear and flanking maskers to the other. For both presentation configurations, thresholds were lowest in conditions employing multiple flankers that were spectrally close to the signal in frequency. This result was interpreted as evidence that relative spectral proximity of the on-signal and flanking maskers is important for CMR, even under conditions free from within-channel effects. This finding could be related to the observation that stimulus components in the CMR stimulus must be grouped together in order to obtain a masking release (Grose and Hall, 1993). While asynchronous gating may interfere with CMR by disrupting grouping, spectral proximity of the flanking masker bands may facilitate grouping and therefore increase CMR. This observation is consistent with the idea that the signal frequency effects that are common to both ipsilateral and contralateral flanker presentation modes are related to "true" across-frequency processes.

\section{Contribution of within-channel effects}

It is commonly observed that the CMR obtained with contralateral flanking bands is smaller than that obtained with ipsilateral flanking bands (Buss and Hall, 2008), and this was true of the present data. Comparison of CMR results obtained with ipsilateral and contralateral flanking masker presentation has been used in the past to differentiate peripheral, within-channel effects from more central, acrosschannel effects. The interaction of neighboring maskers in the auditory periphery has been shown to improve thresholds under some conditions in previous CMR experiments (Schooneveldt and Moore, 1987; Verhey et al., 1999). It is unclear, however, exactly how these within-channel effects could have affected performance in the ipsilateral, allcoherent conditions of the present experiments. Each band was separated in frequency from its neighbors by a factor of 1.38 or more, greater than one equivalent rectangular bandwidth of the auditory filter (Glasberg and Moore, 1990), so the masker bands were at least somewhat independently encoded at the periphery. There is some evidence that withinchannel cues that reduce thresholds in the CMR paradigm, such as those based on beating patterns across frequency (Berg, 1996), are minimized for log spaced, as compared to linearly spaced, bands (Grose et al., 2009). In addition to these possible within-channel effects, suppression has been proposed to play a role in reduced thresholds in the presence of coherently modulated maskers presented ipsilateral to the signal (Bacon and Lee, 1997; Oxenham and Plack, 1998; Ernst and Verhey, 2006).

As in CMR, there are also within-channel cues available in profile analysis, although such cues are not thought to dominate performance in the profile analysis paradigm until neighboring components are separated by less than $1 / 3$ of a critical band (Versfeld and Houtsma, 1995). Within-channel effects could also impair performance in profile analysis under some conditions. In the study of Lentz (2007), spectral weights were estimated for six components that were logarithmically spaced between 700 and $4000 \mathrm{~Hz}$, corresponding to a factor of 1.42 in frequency. The task was to discriminate a set of equal-amplitude tones from a set in which the three low-frequency tones were reduced in amplitude relative to the three high-frequency tones. Level jitter applied to each tone allowed calculation of decision weights associated with each frequency region of the stimulus. Weighting efficiency fell with increasing stimulus level, a result interpreted in terms of upward spread of excitation and masking.

Several published studies include data on the effects of dichotic presentation of profile analysis stimuli that might speak to the role of within-channel effects. However, it has been argued that the results do not reflect "real" profile analysis because dichotic presentation tends to result in the stimulus being perceived as two sound streams (Green and Kidd, 1983). As for CMR, it has been argued that all components of a profile analysis stimulus must be grouped together in order to benefit fully from across-channel cues (Green and Dai, 1992; Hill and Bailey, 1997), and this grouping may be disrupted by dichotic presentation. While similar segregation effects may play a role for dichotic CMR 
under some conditions, coherent modulation of continuously presented stimuli may minimize this effect by providing common envelope cues that bind components in each ear into a single auditory object (Buss and Hall, 2008). It seems likely that this was the case in the present paradigm, as evidenced by subjective impressions as well as the finding of a masking release with contralateral masker presentation in both the present study and comparable conditions of Fantini et al. (1993). To the extent that the all-coherent data of the present study reflect a reliance on dynamic spectral profile cues, the grouping effect of coherent modulation across frequency (Bregman et al., 1985) could increase the probability of obtaining a masking release based on dynamic spectral cues.

In Experiments 1 and 2, the signal frequency associated with best performance in the all-coherent masker condition depended on both signal type and whether or not flanking maskers were presented contralateral to the signal. While this result is broadly consistent with a role for peripheral effects, this interpretation is inconsistent with the profile analysis data of Bernstein and Green (1988). That study reported thresholds in a profile analysis paradigm for logarithmically spaced tones spanning the range 200 to $5000 \mathrm{~Hz}$, varying the number of tones distributed across that range. Greater spectral density would be associated with reduced spectral resolution and increased within-channel effects. A bowl threshold pattern was obtained for a wide range of spectral densities, with a minimum near $1150 \mathrm{~Hz}$ in all cases. That is, the signal frequency associated with best performance was unaffected by spectral proximity of flanking stimulus components. This result is inconsistent with the idea that the availability of within-channel cues is responsible for the different values of fmin for a narrowband noise signal in ipsilateral and contralateral masker conditions. Therefore, there may be other factors besides within-channel effects determining the different signal frequency effects across conditions.

\section{Other peripheral effects}

Whereas within-channel effects may play a role in the frequency associated with best performance ( $f \mathrm{~min})$, it is also possible that the effects of signal type might be explained in terms of shifts in peak excitation as a function of stimulus level. It has been observed that the peak of excitation along the basilar membrane is level dependent under some conditions, an effect attributed to active mechanisms in the cochlea (Ruggero et al., 1997). Moore et al. (2002) demonstrated the psychophysical consequence of this shift in humans. In that study the masker was a $6.5 \mathrm{kHz}$ tone, and thresholds were measured for a forward-masked pure-tone signal over a range of signal frequencies. As signal delay was increased, the signal frequency associated with the maximum forward masking shifted up, an effect interpreted as evidence that the peak excitation associated with the high-level masker was shifted basally relative to the excitation associated with the lower-level signal. While increasing level is associated with a basal-ward shift at $6.5 \mathrm{kHz}$, this effect appears to be frequency specific, with little or no evidence of such a shift at lower frequencies (1-2 kHz; Carney et al., 1999; Moore and Glasberg, 2003).

It is hypothesized here that a basal-ward shift in highfrequency excitation at high presentation levels could contribute to the different all-coherent threshold minima associated with the two signal types. As illustrated in Fig. 1, cues reflecting addition of a tonal signal to a narrowband noise masker occur primarily during masker envelope minima, a characteristic associated with "listening in the dips" for traditional CMR stimuli. In contrast, the cues resulting from addition of a narrowband noise signal to a narrowband noise masker are consistent as a function of time, and are present during both minima and maxima of the masker envelope. One consequence of this difference in the temporal distribution of signal cues is that the spectral peak associated with the tonal signal occurs when the instantaneous stimulus level is low, whereas the peak associated with the narrowband noise signal is present at both low and high instantaneous stimulus levels. Therefore, level could play a larger role in frequency effects for narrowband than tonal signals. Leveldependent shifts in high-frequency excitation could elevate high-frequency narrowband signal thresholds in two ways. First, level effects could hurt performance by shifting signal excitation into a region of reduced sensitivity to spectral cues. Second, dynamic changes in place of transduction of high-frequency energy could themselves interfere with detection, perhaps related to the detrimental effects of stimulus frequency uncertainty (Gockel and Colonius, 1997). It is unclear how a shift in peak excitation could produce different results for ipsilateral and contralateral flanking masker presentation. One possibility is that ipsilateral flanking maskers limit off-frequency listening, whereas contralateral flanking masker presentation increases the number of auditory channels carrying information about the signal.

Although the pattern of all-coherent thresholds differed for the tonal and narrowband noise signals at high frequencies, the pattern of thresholds was similar at low frequencies. This is qualitatively consistent with level-dependent differences in peak excitation at high but not low frequencies (Moore et al., 2002; Moore and Glasberg, 2003). Leveldependent shifts in peak excitation could affect the use of spectral cues for stimuli in which level changes within the listening interval (as in CMR) and for stimuli in which level changes across intervals (as in profile analysis).

\section{E. Relation to profile analysis}

The processes responsible for the bowl shape in the classic profile analysis paradigm employing stationary stimuli are unknown despite numerous demonstrations of that pattern (Green and Mason, 1985; Bernstein and Green, 1987; Green et al., 1987). Early on in the study of profile analysis, it was noted that the bowl resembles audibility (absolute threshold) and equal loudness curves. However, it has been reported that presenting component tones at equal levels relative to threshold does not change the bowl pattern (Green and Berg, 1991).

The bowl in profile analysis has been characterized as reflecting a combination of relative and absolute frequency 
effects, with an increasing role of absolute frequency for high-frequency stimuli (Green and Mason, 1985; Green et al., 1987). Based on data of the present report, it is hypothesized that level rove may impair sensitivity at high frequencies, perhaps due to a combination of place-based absolute frequency effects and shifts in peak excitation related to level. This is the topic of ongoing research, but there is some evidence in the literature for particularly detrimental effects of level rove on spectral shape discrimination above 1000 Hz. Moore et al. (1989) measured level discrimination thresholds for a spectral peak at either 1000 or $8000 \mathrm{~Hz}$. Whereas thresholds were poorer at 8000 than $1000 \mathrm{~Hz}$, introducing a level rove accentuated this frequency effect, having a larger disruptive effect at 8000 than $1000 \mathrm{~Hz}$. These findings are generally consistent with the idea that level variability across intervals interferes with spectral shape discrimination by way of frequency-specific level effects on place of excitation.

\section{SUMMARY}

The present set of experiments demonstrated nonmonotonic effects of signal frequency for signal detection in a comodulated masker complex. At low frequencies, thresholds tended to improve with increasing signal frequency, but at high frequencies that trend was reversed. This basic result is analogous to signal frequency effects observed in profile analysis, described as the profile bowl. The frequency associated with the lowest threshold-the minimum of the bowl-depended on signal type in diotic and ipsilateral masking conditions. The minimum was lower in frequency when the signal was a copy of the narrowband noise masker to which it was added than when it was a tone. Contralateral flanking masker presentation resulted in a higher-frequency minimum than diotic or ipsilateral presentation for both signal types.

Signal frequency effects in comodulated masker conditions were likely due to a combination of relative and absolute frequency effects. Some aspects of the data suggest that relative frequency effects may dominate the falling portion of the bowl (below the minimum), and absolute frequency effects may dominate the rising portion (above the minimum). It was hypothesized that absolute frequency effects in conjunction with effects of level on peak excitation at high frequencies may be responsible for the effects of signal type and ear of flanking masker presentation.

Overall, these results are consistent with the hypothesis that dynamic spectral cues play an important role in the beneficial effects of coherent masker modulation on signal detection, with the caveat that these effects appear to be sensitive to differences in typical CMR and profile analysis stimuli.

\section{ACKNOWLEDGMENTS}

This work was supported by the NIH NIDCD Grant No. RO1-DC007391. Joseph Hall, John Grose, Brian Moore, and two anonymous reviewers provided helpful comments on this work. Tara Steplowski assisted in manuscript preparation.
${ }^{1}$ Different step sizes were used for the two signal types in order to accommodate the different effects of in-phase and random-phase addition on overall level.

${ }^{2}$ For the purpose of data fits, observers' thresholds in the narrowband noise signal conditions $(\mathrm{nb})$ were represented as $n b-50$. That is, the signal threshold was represented as level in $\mathrm{dB}$ re on-signal masker, which in the present experiment was $50 \mathrm{~dB}$ SPL. To maintain comparable units, thresholds in the tonal signal conditions $(t)$ were represented as if the tone were added in phase to the masker. To accomplish this, the level of the signalplus-masker was computed as $x=10 \log \left(10^{t / 10}+10^{50 / 10}\right)$. The masker level was then subtracted in amplitude, with $y=20 \log \left(10^{x / 20}-10^{50 / 20}\right)$. The result, $y$, is the level of a narrowband noise signal associated with the same change in level as the tonal signal. Tonal thresholds were then represented as $y-50$.

ANSI (2004). ANSI S3.6-2004, American National Standard Specification for Audiometers (American National Standards Institute, New York).

Bacon, S. P., Grimault, N., and Lee, J. (2002). "Spectral integration in bands of modulated or unmodulated noise," J. Acoust. Soc. Am. 112, 219-226.

Bacon, S. P., and Lee, J. (1997). "The modulated-unmodulated difference: Effects of signal frequency and masker modulation depth," J. Acoust. Soc. Am. 101, 3617-3624.

Bacon, S. P., and Smith, M. A. (1996). "Profile analysis with amplitudemodulated nontarget components," J. Acoust. Soc. Am. 99, 1653-1659.

Berg, B. G. (1996). "On the relation between comodulation masking release and temporal modulation transfer functions," J. Acoust. Soc. Am. 100, 1013-1023.

Bernstein, L. R., and Green, D. M. (1987). "The profile-analysis bandwidth,” J. Acoust. Soc. Am. 81, 1888-1895.

Bernstein, L. R., and Green, D. M. (1988). "Detection of changes in spectral shape: Uniform vs. non-uniform background spectra," Hear. Res. 34, 157165.

Bos, C. E., and de Boer, E. (1966). "Masking and discrimination," J. Acoust. Soc. Am. 39, 708-715.

Bregman, A. S., Abramson, J., Doehring, P., and Darwin, C. J. (1985). "Spectral integration based on common amplitude modulation," Percept. Psychophys. 37, 483-493.

Buss, E., Grose, J. H., and Hall, J. W. (2009). "Features of across-frequency envelope coherence critical for comodulation masking release," J. Acoust. Soc. Am. 126, 2455-2466.

Buss, E., and Hall, J. W. (2008). "Factors contributing to comodulation masking release with dichotic maskers," J. Acoust. Soc. Am. 124, 19051908.

Buus, S. (1985). "Release from masking caused by envelope fluctuations," J. Acoust. Soc. Am. 78, 1958-1965.

Buus, S., Zhang, L., and Florentine, M. (1996). "Stimulus-driven, timevarying weights for comodulation masking release," J. Acoust. Soc. Am. 99, 2288-2297.

Carney, L. H., McDuffy, M. J., and Shekhter, I. (1999). "Frequency glides in the impulse responses of auditory-nerve fibers," J. Acoust. Soc. Am. 105, 2384-2391.

Cohen, M. F. (1991). "Comodulation masking release over a three octave range," J. Acoust. Soc. Am. 90, 1381-1384.

Cohen, M. F., and Schubert, E. D. (1987). "Influence of place synchrony on detection of a sinusoid," J. Acoust. Soc. Am. 81, 452-458.

Durlach, N. I., Braida, L. D., and Ito, Y. (1986). "Towards a model for discrimination of broadband signals," J. Acoust. Soc. Am. 80, 63-72.

Eddins, D. A. (2001). "Monaural masking release in random-phase and low-noise noise," J. Acoust. Soc. Am. 109, 1538-1549.

Ellermeier, W. (1996). "Detectability of increments and decrements in spectral profiles," J. Acoust. Soc. Am. 99, 3119-3125.

Ernst, S. M., and Verhey, J. L. (2006). "Role of suppression and retrocochlear processes in comodulation masking release," J. Acoust. Soc. Am. 120, 3843-3852.

Ernst, S. M., and Verhey, J. L. (2008). "Peripheral and central aspects of auditory across-frequency processing," Brain Res. 1220, 246-255.

Fantini, D. A., and Moore, B. C. J. (1994a). "A comparison of the effectiveness of across-channel cues available in comodulation masking release and profile analysis tasks," J. Acoust. Soc. Am. 96, 3451-3462.

Fantini, D. A., and Moore, B. C. J. (1994b). "Profile analysis and comodulation detection differences using narrow bands of noise and their relation to comodulation masking release," J. Acoust. Soc. Am. 95, 2180-2191.

Fantini, D. A., Moore, B. C. J., and Schooneveldt, G. P. (1993). "Comodulation masking release as a function of type of signal, gated or continuous 
masking, monaural or dichotic presentation of flanking bands, and center frequency," J. Acoust. Soc. Am. 93, 2106-2115.

Glasberg, B. R., and Moore, B. C. J. (1990). "Derivation of auditory filter shapes from notched-noise data," Hear. Res. 47, 103-138.

Gockel, H., and Colonius, H. (1997). "Auditory profile analysis: Is there perceptual constancy for spectral shape for stimuli roved in frequency?," $\mathrm{J}$ Acoust. Soc. Am. 102, 2311-2315.

Green, D. M. (1988). Profile Analysis: Auditory Intensity Discrimination (Oxford University Press, New York).

Green, D. M., and Berg, B. G. (1991). "Spectral weights and the profile bowl," Q. J. Exp. Psychol. A 43, 449-458.

Green, D. M., and Dai, H. (1992). "Temporal relations in profile comparisons," in Auditory Physiology and Perception, edited by Y. Cazals, L. Demany, and K. Horner, (Pergamon, Oxford), pp. 471-477.

Green, D. M., and Kidd, G., Jr. (1983). "Further studies of auditory profile analysis," J. Acoust. Soc. Am. 73, 1260-1265.

Green, D. M., and Mason, C. R. (1985). "Auditory profile analysis: Frequency, phase, and Weber's law," J. Acoust. Soc. Am. 77, 1155-1161.

Green, D. M., and Nguyen, Q. T. (1988). "Profile analysis: Detecting dynamic spectral changes," Hear. Res. 32, 147-163.

Green, D. M., Onsan, Z. A., and Forrest, T. G. (1987). "Frequency effects in profile analysis and detecting complex spectral changes," J. Acoust. Soc. Am. 81, 692-699.

Grose, J. H., Buss, E., and Hall, J. W. (2009). "Within- and across-channel factors in the multiband comodulation masking release paradigm," J. Acoust. Soc. Am. 125, 282-293.

Grose, J. H., and Hall, J. W. (1990). "The effect of signal-frequency uncertainty on comodulation masking release," J. Acoust. Soc. Am. 87, 12721277.

Grose, J. H., and Hall, J. W. (1993). "Comodulation masking release: Is comodulation sufficient?," J. Acoust. Soc. Am. 93, 2896-2902.

Grose, J. H., Hall, J. W., Buss, E., and Hatch, D. R. (2005). "Detection of spectrally complex signals in comodulated maskers: Effect of temporal fringe," J. Acoust. Soc. Am. 118, 3774-3782.

Haggard, M. P., Hall, J. W., and Grose, J. H. (1990). "Comodulation masking release as a function of bandwidth and test frequency," J. Acoust. Soc. Am. 88, 113-118.

Hall, J. W. (1986). "The effect of across-frequency differences in masking level on spectro-temporal pattern analysis," J. Acoust. Soc. Am. 79, 781787.

Hall, J. W., and Grose, J. H. (1988). "Comodulation masking release: Evidence for multiple cues," J. Acoust. Soc. Am. 84, 1669-1675.

Hall, J. W., Grose, J. H., and Haggard, M. P. (1988). "Comodulation masking release for multicomponent signals," J. Acoust. Soc. Am. 83, 677-686.

Hall, J. W., Grose, J. H., and Haggard, M. P. (1990). "Effects of flanking band proximity, number, and modulation pattern on comodulation masking release," J. Acoust. Soc. Am. 87, 269-283.

Hall, J. W., Haggard, M. P., and Fernandes, M. A. (1984). "Detection in noise by spectro-temporal pattern analysis," J. Acoust. Soc. Am. 76, 5056.

Hill, N. I., and Bailey, P. J. (1997). "Profile analysis with an asynchronous target: Evidence for auditory grouping," J. Acoust. Soc. Am. 102, 477481.

Langhans, A., and Kohlrausch, A. (1992). "Differences in auditory performance between monaural and dichotic conditions. I: Masking thresholds in frozen noise," J. Acoust. Soc. Am. 91, 3456-3470.

Lentz, J. J. (2005). "Profile analysis: The effects of rove on sparse spectra," J. Acoust. Soc. Am. 118, 2794-2797.

Lentz, J. J. (2006). "Spectral-peak selection in spectral-shape discrimination by normal-hearing and hearing-impaired listeners," J. Acoust. Soc. Am. 120, 945-956.

Lentz, J. J. (2007). "Variation in spectral-shape discrimination weighting functions at different stimulus levels and signal strengths," J. Acoust. Soc. Am. 122, 1702

Levitt, H. (1971). "Transformed up-down methods in psychoacoustics," J. Acoust. Soc. Am. 49, 467-477.

McFadden, D. (1986). "Comodulation masking release: Effects of varying the level, duration, and time delay of the cue band," J. Acoust. Soc. Am. 80, 1658-1667.

Moore, B. C. J., Alcantara, J. I., and Glasberg, B. R. (2002). "Behavioural measurement of level-dependent shifts in the vibration pattern on the basilar membrane," Hear. Res. 163, 101-110.

Moore, B. C. J., and Glasberg, B. R. (1987). "Factors affecting thresholds for sinusoidal signals in narrow-band maskers with fluctuating envelopes," J. Acoust. Soc. Am. 82, 69-79.

Moore, B. C. J., and Glasberg, B. R. (2003). "Behavioural measurement of level-dependent shifts in the vibration pattern on the basilar membrane at 1 and $2 \mathrm{kHz}$," Hear. Res. 175, 66-74.

Moore, B. C. J., Glasberg, B. R., and Schooneveldt, G. P. (1990). "Acrosschannel masking and comodulation masking release," J. Acoust. Soc. Am. 87, 1683-1694

Moore, B. C. J., Oldfield, S. R., and Dooley, G. J. (1989). "Detection and discrimination of spectral peaks and notches at 1 and $8 \mathrm{kHz}$," J. Acoust. Soc. Am. 85, 820-836.

Oxenham, A. J., and Plack, C. J. (1998). "Suppression and the upward spread of masking," J. Acoust. Soc. Am. 104, 3500-3510.

Richards, V. M. (1987). "Monaural envelope correlation perception," J. Acoust. Soc. Am. 82, 1621-1630.

Richards, V. M., and Lentz, J. J. (1998). "Sensitivity to changes in level and envelope patterns across frequency," J. Acoust. Soc. Am. 104, 3019-3029.

Ruggero, M. A., Rich, N. C., Recio, A., Narayan, S. S., and Robles, L. (1997). "Basilar-membrane responses to tones at the base of the chinchilla cochlea," J. Acoust. Soc. Am. 101, 2151-2163.

Schooneveldt, G. P., and Moore, B. C. J. (1987). "Comodulation masking release (CMR): Effects of signal frequency, flanking-band frequency, masker bandwidth, flanking-band level, and monotic versus dichotic presentation of the flanking band," J. Acoust. Soc. Am. 82, 1944-1956.

Schooneveldt, G. P., and Moore, B. C. J. (1989). "Comodulation masking release for various monaural and binaural combinations of the signal, onfrequency, and flanking bands," J. Acoust. Soc. Am. 85, 262-272.

van de Par, S., and Kohlrausch, A. (1998). "Comparison of monaural (CMR) and binaural (BMLD) masking release," J. Acoust. Soc. Am. 103, 1573-1579.

Verhey, J. L., Dau, T., and Kollmeier, B. (1999). "Within-channel cues in comodulation masking release (CMR): Experiments and model predictions using a modulation-filterbank model," J. Acoust. Soc. Am. 106, 2733-2745.

Versfeld, N. J., and Houtsma, A. J. (1995). "Discrimination of changes in the spectral shape of two-tone complexes," J. Acoust. Soc. Am. 98, 807816. 